\title{
A Crank-Nicolson finite difference scheme for the Riesz space fractional-order parabolic-type sine-Gordon equation
}

\author{
Yanjie Zhou' and Zhendong Luo ${ }^{2 *}$
}

"Correspondence: zhdluo@163.com ${ }^{2}$ School of Mathematics and Physics, North China Electric Power University, Beijing, China Full list of author information is available at the end of the article

\begin{abstract}
In this paper, we focus on the study of the Crank-Nicolson finite difference (CNFD) scheme for the Riesz space fractional-order parabolic-type sine-Gordon equation (RSFOPTSGE). For this purpose, we first establish the CNFD scheme for RSFOPTSGE. Then, we discuss the existence, uniqueness, stability, and convergence of the CNFD solutions. Finally, we supply a numerical experiment to validate the correctness of theoretical results. This indicates that the CNFD scheme is very effective for solving RSFOPTSGE.

MSC: 34K28; 35R11;65M12

Keywords: Crank-Nicolson finite difference scheme; Riesz space fractional-order parabolic-type sine-Gordon equation; Existence, stability, and convergence; Numerical experiment
\end{abstract}

\section{Introduction}

The fractional-order differential equations have become a research hot-spot in science and engineering in recent years (see, e.g., $[1,2])$. Unfortunately, there are very few researches of numerical solutions for the standard Riesz space fractional-order sine-Gordon equation (see, e.g., [3-5]). However, the Riesz space fractional-order parabolic-type sine-Gordon equation (RSFOPTSGE) with the first-order time derivative is not studied. Therefore, this paper mainly focuses on the study of the Crank-Nicolson finite difference (CNFD) scheme for RSFOPTSGE with first-order time derivative.

For convenience and without loss of generality, we consider the following RSFOPTSGEs on the bounded domain $[0, T] \times[0, L]$ :

$$
\begin{aligned}
& \frac{\partial u(t, x)}{\partial t}=K \frac{\partial^{\alpha} u(t, x)}{\partial|\alpha|^{\alpha}}+\sin (u(t, x)), \quad(t, x) \in(0, T) \times(0, L), \\
& u(t, 0)=u(t, L)=g(t), \quad t \in(0, T), \\
& u(0, x)=\varphi(x), \quad x \in(0, L),
\end{aligned}
$$

where $K$ is the dispersion coefficient, $1<\alpha \leq 2, g(t)$ is a given boundary value function, $\varphi(t, x)$ is a given initial function, and $\partial^{\alpha} u(t, x) / \partial|\alpha|^{\alpha}$ is known as the Riesz space fractional-

(c) The Author(s) 2018. This article is distributed under the terms of the Creative Commons Attribution 4.0 International License (http://creativecommons.org/licenses/by/4.0/), which permits unrestricted use, distribution, and reproduction in any medium, provided you give appropriate credit to the original author(s) and the source, provide a link to the Creative Commons license, and indicate if changes were made. 
order derivative defined by

$$
\frac{\partial^{\alpha} u(t, x)}{\partial|\alpha|^{\alpha}}=S(\alpha) \frac{\partial^{2}}{\partial x^{2}}\left(\int_{0}^{x}(x-y)^{1-\alpha} u(t, y) \mathrm{d} y+\int_{x}^{L}(y-x)^{1-\alpha} u(t, y) \mathrm{d} y\right)
$$

where $S(\alpha)=-\left[2 \cos \left(\frac{\alpha \pi}{2}\right) \Gamma(2-\alpha)\right]^{-1}$, and $\Gamma(\cdot)$ is the Euler gamma function. For convenience and without loss of generality, we further assume that $g(t)=0$.

The RSFOPTSGEs (1)-(3), which is substantially a parabolic-type Riesz space fractionalorder partial differential equation with nonlinear source term $\sin (u)$, just as the standard Riesz space fractional-order differential equations in [3-5], also hold very important physical background, such as phenomena in seepage hydraulics groundwater hydraulics, groundwater dynamics, and fluid dynamics in porous media (see, e.g., [1-5]). However, they usually have no analytic solution, so that we mainly depend on numerical solutions (see, e.g., $[4,5])$. Some finite difference (FD) schemes for the standard Riesz space fractional-order differential equations have been established in $[4,5]$, but there is no any theoretical analysis about the stability and convergence and error estimates for their FD solutions. Therefore, in this paper, we establish a CNFD scheme for the RSFOPTSGEs (1)-(3) and provide theoretical analysis about the stability and convergence and error estimates for the CNFD solutions. We also use a numerical experiment to validate the correctness of the theoretical results.

Though there have been many studies for the standard sine-Gordon equations (see, e.g., [6-10]), because RSFOPTSGEs not only include a first-order time derivative and the Riesz space fractional-order derivative, but also contain a nonlinear source term $\sin (u)$, the establishment of the CNFD scheme for the RSFOPTSGEs (1)-(3) and theoretical analysis of the stability and convergence and error estimates for the CNFD solutions are faced with more difficulties and require more techniques than the standard sine-Gordon equations. However, RSFOPTSGEs with first-order time derivative have some special applications; for example, they can be used to describe the sin-waves attenuation phenomenon. Therefore our work is interesting, is different from the existing others, and is a development and improvement of the latter.

The rest of this paper is arranged as follows. In Sect. 2, we first establish the CNFD scheme for the RSFOPTSGEs (1)-(3). Then, in Sect. 3, we discuss the existence, stability, and convergence of the CNFD solutions for the RSFOPTSGEs (1)-(3). Next, in Sect. 4, we use a numerical experiment to validate the correctness of the theoretical analysis and show that the CNFD scheme is very efficient for solving RSFOPTSGEs. Finally, Sect. 5 provides the main conclusions and discussions.

\section{Establishment of the CNFD scheme for RSFOPTSGEs}

Let $N$ and $M$ be two positive integers, let $\tau=T / N$ be the time step-size, and let $h=L / M$ be the spatial step-size. Thus, the CNFD scheme with the predictor-corrector for the RSFOPTSGEs (1)-(3) is stated as follows:

$$
\bar{u}_{i}^{n}=u_{i}^{n-1}+\gamma\left[\sum_{k=0}^{i+1} \omega_{k}^{(\alpha)} u_{i-k+1}^{n-1}+\sum_{k=0}^{M-i+1} \omega_{k}^{(\alpha)} u_{i+k-1}^{n-1}\right]+\tau \sin \left(u_{i}^{n-1}\right)
$$




$$
\begin{aligned}
u_{i}^{n}= & u_{i}^{n-1}+\gamma\left[\sum_{k=0}^{i+1} \omega_{k}^{(\alpha)} u_{i-k+1}^{n-1}+\sum_{k=0}^{M-i+1} \omega_{k}^{(\alpha)} u_{i+k-1}^{n-1}\right]+\frac{\tau}{2} \sin \left(u_{i}^{n-1}\right) \\
& +\gamma\left[\sum_{k=0}^{i+1} \omega_{k}^{(\alpha)} \bar{u}_{i-k+1}^{n-1}+\sum_{k=0}^{M-i+1} \omega_{k}^{(\alpha)} \bar{u}_{i+k-1}^{n-1}\right]+\frac{\tau}{2} \sin \left(\bar{u}_{i}^{n-1}\right),
\end{aligned}
$$

where $u_{i}^{n}$ 's are approximate solutions of $u\left(t_{n}, x_{i}\right)(i=1,2, \ldots, M), \gamma=-\tau K /\left[2 h^{\alpha} \cos (\alpha \pi / 2)\right]$, $\omega_{0}^{(\alpha)}=\alpha g_{0}^{(\alpha)} / 2, \omega_{k}^{(\alpha)}=\alpha g_{k}^{(\alpha)} / 2+(2-\alpha) g_{k-1}^{(\alpha)} / 2, g_{0}^{(\alpha)}=1, g_{k}^{(\alpha)}=[1-(1+\alpha) / k] g_{k-1}^{(\alpha)}(k=1,2, \ldots)$.

The sequences $\left\{\omega_{k}^{(\alpha)}\right\}_{k=0}^{\infty}$ and $\left\{g_{k}^{(\alpha)}\right\}_{k=0}^{\infty}$ have the following properties (see, e.g., [2, 9]).

Lemma 1 When $1<\alpha \leq 2$, the sequences $\left\{\omega_{k}^{(\alpha)}\right\}_{k=0}^{\infty}$ and $\left\{g_{k}^{(\alpha)}\right\}_{k=0}^{\infty}$ satisfy

$$
\begin{aligned}
& g_{0}^{(\alpha)}=1, \quad g_{1}^{(\alpha)}=-\alpha, \quad g_{2}^{(\alpha)}=\alpha(\alpha-1) / 2>0, \\
& 1 \geq g_{2}^{(\alpha)} \geq g_{3}^{(\alpha)} \geq \cdots \geq 0, \quad \sum_{k=0}^{\infty} g_{k}^{(\alpha)}=0, \quad \sum_{k=0}^{m} g_{k}^{(\alpha)}<0 \quad(m \geq 1) ; \\
& \omega_{0}^{(\alpha)}=\alpha / 2, \quad \omega_{1}^{(\alpha)}=\left(2-\alpha-\alpha^{2}\right) / 2<0, \quad \omega_{2}^{(\alpha)}=\alpha\left(\alpha^{2}+\alpha-4\right) / 4>0, \\
& 1 \geq \omega_{3}^{(\alpha)} \geq \omega_{4}^{(\alpha)} \geq \cdots \geq 0, \quad \sum_{k=0}^{\infty} \omega_{k}^{(\alpha)}=0, \quad \sum_{k=0}^{m} \omega_{k}^{(\alpha)}<0 \quad(m \geq 2) .
\end{aligned}
$$

Set

$$
\begin{aligned}
\mathbf{A} & =\left[\begin{array}{cccccc}
\omega_{1}^{(\alpha)} & \omega_{0}^{(\alpha)} & 0 & \cdots & 0 & 0 \\
\omega_{2}^{(\alpha)} & \omega_{1}^{(\alpha)} & \omega_{0}^{(\alpha)} & \ldots & 0 & 0 \\
\omega_{3}^{(\alpha)} & \omega_{2}^{(\alpha)} & \omega_{1}^{(\alpha)} & \cdots & 0 & 0 \\
\vdots & \vdots & \vdots & \ddots & \vdots & \vdots \\
\omega_{M-2}^{(\alpha)} & \omega_{M-3}^{(\alpha)} & \omega_{M-4}^{(\alpha)} & \cdots & \omega_{1}^{(\alpha)} & \omega_{0}^{(\alpha)} \\
\omega_{M-1}^{(\alpha)} & \omega_{M-2}^{(\alpha)} & \omega_{M-3}^{(\alpha)} & \cdots & \omega_{2}^{(\alpha)} & \omega_{1}^{(\alpha)}
\end{array}\right], \quad \mathbf{F}\left(\mathbf{U}^{n-1}\right)=\left[\begin{array}{c}
\sin \left(u_{1}^{n-1}\right) \\
\sin \left(u_{2}^{n-1}\right) \\
\sin \left(u_{3}^{n-1}\right) \\
\vdots \\
\sin \left(u_{M-2}^{n-1}\right) \\
\sin \left(u_{M-1}^{n-1}\right)
\end{array}\right], \\
\mathbf{U}^{n} & =\left[\begin{array}{ll}
\left.u_{1}^{n}, u_{2}^{n}, \ldots, u_{M-2}^{n}, u_{M-1}^{n}\right]^{T}, \\
\mathbf{D}
\end{array}=-\gamma\left[\mathbf{A}+\mathbf{A}^{T}\right] .\right.
\end{aligned}
$$

Thus, the CNFD scheme (5)-(6) can rewritten in the following matrix form:

$$
\begin{aligned}
& \overline{\mathbf{U}}^{n}=\mathbf{U}^{n-1}+\mathbf{D} \mathbf{U}^{n-1}+\tau \mathbf{F}\left(\mathbf{U}^{n-1}\right), \\
& \mathbf{U}^{n}=\mathbf{U}^{n-1}+\frac{1}{2} \mathbf{D}\left(\mathbf{U}^{n-1}+\overline{\mathbf{U}}^{n-1}\right)+\frac{\tau}{2}\left[\mathbf{F}\left(\mathbf{U}^{n-1}\right)+\mathbf{F}\left(\overline{\mathbf{U}}^{n-1}\right)\right] .
\end{aligned}
$$

Further, the vector form CNFD scheme (7)-(8) can be simplified as follows:

$$
\begin{aligned}
\mathbf{U}^{n}= & \mathbf{U}^{n-1}+\frac{1}{2} \mathbf{D}\left(2 \mathbf{U}^{n-1}+\mathbf{D} \mathbf{U}^{n-1}+\tau \mathbf{F}\left(\mathbf{U}^{n-1}\right)\right) \\
& +\frac{\tau}{2}\left[\mathbf{F}\left(\mathbf{U}^{n-1}\right)+\mathbf{F}\left(\mathbf{U}^{n-1}+\mathbf{D} \mathbf{U}^{n-1}+\tau \mathbf{F}\left(\mathbf{U}^{n-1}\right)\right)\right], \quad n=1,2, \ldots, N,
\end{aligned}
$$

subject to the initial condition

$$
\mathbf{U}^{0}=\left[u_{1}^{0}, u_{2}^{0}, \ldots, u_{M-2}^{0}, u_{M-1}^{0}\right]^{T}, \quad u_{i}^{0}=\varphi(0, i h), i=1,2, \ldots, M-1 .
$$




\section{The existence, stability, and convergence of the CNFD solutions}

Obviously, the vector form CNFD scheme (9)-(10) has a unique series of solution vectors $\left\{\mathbf{U}^{n}\right\}_{n=1}^{N}$. To analyze the stability and convergence of the CNFD solutions, we review the max-norms of matrices and vectors (for more details, see [11]), which are, respectively, defined by $\|\tilde{\mathbf{A}}\|_{\infty}=\max _{1 \leq i \leq m} \sum_{j=1}^{m}\left|a_{i, j}\right|\left(\right.$ for any matrix $\tilde{\mathbf{A}}=\left(a_{i, j}\right)_{m \times m} \in \mathbb{R}^{m} \times \mathbb{R}^{m}$ ) and $\left\|\mathbf{U}^{i}\right\|_{\infty}=\max _{1 \leq j \leq m}\left|u_{j}^{i}\right|\left(\right.$ for any $\left.\mathbf{U}^{i}=\left(u_{1}^{i}, u_{2}^{i}, \ldots, u_{m}^{i}\right)^{T} \in \mathbb{R}^{m}\right)$.

We hold the following result on the stability and convergence of the series for the CNFD solutions $\left\{\mathbf{U}^{n}\right\}_{n=1}^{N}$.

Theorem 2 When $\|\mathbf{I}+\mathbf{D}\|_{\infty} \leq 1$, the series of solutions $\left\{\mathbf{U}^{n}\right\}_{n=1}^{N}$ for the CNFD scheme (9)(10) is stable and convergent. Furthermore, the errors between the series of the CNFD solutions $\left\{\mathbf{U}^{n}\right\}_{n=1}^{N}$ for the CNFD scheme (9)-(10) and $\mathbf{U}_{a}^{n}=\left[u\left(t_{n}, x_{1}\right), u\left(t_{n}, x_{2}\right), \ldots, u\left(t_{n}, x_{M-1}\right)\right]^{T}$ $(n=1,2, \ldots, N)$ formed by the analytical solution for the RSFOPTSGEs (1)-(3) satisfy the estimate

$$
\left\|\mathbf{U}^{n}-\mathbf{U}_{a}^{n}\right\|_{\infty}=O\left(\tau^{2}, h^{2}\right), \quad n=1,2, \ldots, N,
$$

where I represents the unit matrix.

Proof

(1) The stability and convergence of the CNFD solutions $\left\{\mathbf{U}^{n}\right\}_{n=1}^{N}$

When $\|\mathbf{I}+\mathbf{D}\|_{\infty} \leq 1$, we have

$$
\left\|\mathbf{I}+\mathbf{D}+\frac{1}{2} \mathbf{D}^{2}\right\|_{\infty}=\left\|\frac{1}{2}\left[\mathbf{I}+(\mathbf{I}+\mathbf{D})^{2}\right]\right\|_{\infty} \leq \frac{1}{2}\left(1+\|\mathbf{I}+\mathbf{D}\|_{\infty}\right) \leq 1 .
$$

By the differential mean value theorem we have

$$
\begin{aligned}
& \left\|\mathbf{F}\left(\mathbf{U}\left(t_{i}\right)\right)-\mathbf{F}\left(\mathbf{U}^{i}\right)\right\|_{\infty} \leq\left\|\mathbf{U}\left(t_{i}\right)-\mathbf{U}^{i}\right\|_{\infty}, \\
& \left\|\mathbf{F}\left(\mathbf{U}^{i}\right)\right\|_{\infty} \leq\left\|\mathbf{U}^{i}\right\|_{\infty} .
\end{aligned}
$$

Hence, by (12)-(14) from (9) we have

$$
\begin{aligned}
\left\|\mathbf{U}^{n}\right\|_{\infty} \leq & \left\|\mathbf{U}^{n-1}+\frac{1}{2} \mathbf{D}\left(2 \mathbf{U}^{n-1}+\mathbf{D} \mathbf{U}^{n-1}\right)\right\|_{\infty}+\left\|\frac{\tau}{2} \mathbf{D F}\left(\mathbf{U}^{n-1}\right)\right\|_{\infty} \\
& +\left\|\frac{\tau}{2}\left[\mathbf{F}\left(\mathbf{U}^{n-1}\right)+\mathbf{F}\left(\mathbf{U}^{n-1}+\mathbf{D} \mathbf{U}^{n-1}+\tau \mathbf{F}\left(\mathbf{U}^{n-1}\right)\right)\right]\right\|_{\infty} \\
\leq & \left\|\mathbf{I}+\mathbf{D}+\frac{1}{2} \mathbf{D}^{2}\right\|_{\infty}\left\|\mathbf{U}^{n-1}\right\|_{\infty}+\frac{\tau}{2}\|\mathbf{D}\|_{\infty}\left\|\mathbf{F}\left(\mathbf{U}^{n-1}\right)\right\|_{\infty} \\
& +\frac{\tau}{2}\left[\left\|\mathbf{F}\left(\mathbf{U}^{n-1}\right)\right\|_{\infty}+\left\|\mathbf{F}\left(\mathbf{U}^{n-1}+\mathbf{D} \mathbf{U}^{n-1}+\tau \mathbf{F}\left(\mathbf{U}^{n-1}\right)\right)\right\|_{\infty}\right] \\
\leq & \left\|\mathbf{U}^{n-1}\right\|_{\infty}+\frac{\tau}{2}\|\mathbf{D}\|_{\infty}\left\|\mathbf{U}^{n-1}\right\|_{\infty}+\frac{\tau}{2}\left\|\mathbf{U}^{n-1}\right\|_{\infty} \\
& +\frac{\tau}{2}\left\|(\mathbf{I}+\mathbf{D}) \mathbf{U}^{n-1}+\tau \mathbf{F}\left(\mathbf{U}^{n-1}\right)\right\|_{\infty} \\
\leq & \left\|\mathbf{U}^{n-1}\right\|_{\infty}+\frac{\tau}{2}\|\mathbf{D}\|_{\infty}\left\|\mathbf{U}^{n-1}\right\|_{\infty}+\tau\left\|\mathbf{U}^{n-1}\right\|_{\infty}+\frac{\tau^{2}}{2}\left\|\mathbf{U}^{n-1}\right\|_{\infty} \\
= & (1+\beta \tau)\left\|\mathbf{U}^{n-1}\right\|_{\infty}, \quad n=1,2, \ldots, N,
\end{aligned}
$$


where $\beta=1+\tau / 2+\|\mathbf{D}\|_{\infty} / 2$. Because $\|\mathbf{D}\|_{\infty}=\|\mathbf{I}+\mathbf{D}-\mathbf{I}\|_{\infty} \leq\|\mathbf{I}+\mathbf{D}\|_{\infty}+\|\mathbf{I}\|_{\infty} \leq 2$, $\beta \leq 2+\tau / 2 \leq 2+\tau$. Thus, by iterating (15) we have

$$
\begin{aligned}
\left\|\mathbf{U}^{n}\right\|_{\infty} & \leq(1+\beta \tau)^{n}\left\|\mathbf{U}^{0}\right\|_{\infty} \leq\left\|\mathbf{U}^{0}\right\|_{\infty} \exp [(2+\tau) n \tau] \\
& \leq\left\|\mathbf{U}^{0}\right\|_{\infty} \exp [(2+\tau) T], \quad n=1,2, \ldots, N
\end{aligned}
$$

which shows that the CNFD solutions $\left\{\mathbf{U}^{n}\right\}_{n=1}^{N}$ are stable and convergent according to the Lax stability theorem (see [11]).

(2) The error estimates (11) of the CNFD solutions $\left\{\mathbf{U}^{n}\right\}_{n=1}^{N}$

Let $\mathbf{U}_{a}^{n}=\left[u\left(t_{n}, x_{1}\right), u\left(t_{n}, x_{2}\right), \ldots, u\left(t_{n}, x_{M-1}\right)\right]^{T}$, as mentioned before, be formed by the analytical solution for the RSFOPTSGEs (1)-(3), and let $\mathbf{e}_{n}=\mathbf{U}^{n}-\mathbf{U}_{d}^{n}$. By subtracting (9) from RSFOPTSGEs (1)-(3), by Taylor's formula (see, e.g., [11]) we have

$$
\begin{aligned}
\mathbf{e}_{n}= & \mathbf{e}_{n-1}+\frac{1}{2} \mathbf{D}\left(2 \mathbf{e}_{n-1}+\mathbf{D} \mathbf{e}_{n-1}+\tau \mathbf{F}\left(\mathbf{U}^{n-1}\right)-\tau \mathbf{F}\left(\mathbf{U}_{a}^{n-1}\right)\right)+\frac{\tau}{2}\left[\mathbf{F}\left(\mathbf{U}^{n-1}\right)\right. \\
& +\mathbf{F}\left(\mathbf{U}^{n-1}+\mathbf{D} \mathbf{U}^{n-1}+\tau \mathbf{F}\left(\mathbf{U}^{n-1}\right)\right) \\
& \left.-\mathbf{F}\left(\mathbf{U}_{a}^{n-1}\right)-\mathbf{F}\left(\mathbf{U}_{a}^{n-1}+\mathbf{D} \mathbf{U}_{a}^{n-1}+\tau \mathbf{F}\left(\mathbf{U}_{a}^{n-1}\right)\right)\right]+O\left(\tau^{3}+\tau h^{2}\right) \\
= & \frac{\tau}{2}\left[\mathbf{F}\left(\mathbf{U}^{n-1}+\mathbf{D} \mathbf{U}^{n-1}+\tau \mathbf{F}\left(\mathbf{U}^{n-1}\right)\right)-\mathbf{F}\left(\mathbf{U}_{a}^{n-1}+\mathbf{D} \mathbf{U}_{a}^{n-1}+\tau \mathbf{F}\left(\mathbf{U}_{a}^{n-1}\right)\right)\right] \\
& +\left(\mathbf{I}+\mathbf{D}+\frac{1}{2} \mathbf{D}^{2}\right) \mathbf{e}_{n-1}+\frac{\tau}{2}(\mathbf{I}+\mathbf{D})\left[\mathbf{F}\left(\mathbf{U}^{n-1}\right)-\mathbf{F}\left(\mathbf{U}_{a}^{n-1}\right)\right]+O\left(\tau^{3}+\tau h^{2}\right) .
\end{aligned}
$$

By using (13)-(14), $\|\mathbf{I}+\mathbf{D}\|_{\infty} \leq 1$, and $\left\|\mathbf{I}+\mathbf{D}+\mathbf{D}^{2} / 2\right\|_{\infty} \leq 1$, from (17) we have

$$
\begin{aligned}
\left\|\mathbf{e}_{n}\right\|_{\infty} \leq & \left\|\mathbf{I}+\mathbf{D}+\frac{1}{2} \mathbf{D}^{2}\right\|_{\infty}\left\|\mathbf{e}_{n-1}\right\|_{\infty}+\frac{\tau}{2}\|\mathbf{D}\|_{\infty}\left\|\mathbf{e}_{n-1}\right\|_{\infty} \\
& +\frac{\tau}{2}\left[\left\|\mathbf{e}_{n-1}\right\|_{\infty}+\|\mathbf{I}+\mathbf{D}\|_{\infty}\left\|\mathbf{e}_{n-1}\right\|_{\infty}+\tau\left\|\mathbf{e}_{n-1}\right\|_{\infty}\right]+C\left(\tau^{3}+\tau h^{2}\right) \\
\leq & (1+\beta \tau)\left\|\mathbf{e}_{n-1}\right\|_{\infty}+C\left(\tau^{3}+\tau h^{2}\right) \\
\leq & \left(1+2 \tau+\tau^{2}\right)\left\|\mathbf{e}_{n-1}\right\|_{\infty}+C\left(\tau^{3}+\tau h^{2}\right),
\end{aligned}
$$

where $C$ is a generic positive constant. Thus, iterating (18) and using $\mathbf{e}_{0}=\mathbf{0}$, we have

$$
\begin{aligned}
\left\|\mathbf{e}_{n}\right\|_{\infty} & \leq\left(1+2 \tau+\tau^{2}\right)^{n}\left\|\mathbf{e}_{0}\right\|_{\infty}+C n\left(\tau^{3}+\tau h^{2}\right) \\
& \leq C T\left(\tau^{2}+h^{2}\right) \exp [n \tau(2+\tau)] \\
& \leq C T\left(\tau^{2}+h^{2}\right) \exp [T(2+\tau)], \quad n=1,2, \ldots, N .
\end{aligned}
$$

This attains (11) and accomplishes the demonstration of Theorem 2.

Remark 3 From Lemma 1 we easily see that the condition $\|\mathbf{I}+\mathbf{D}\|_{\infty} \leq 1$ is reasonable.

\section{Numerical experiment}

In this section, we give a numerical experiment to validate the correctness of the theoretical results of the CNFD scheme for the RSFOPTSGEs (1)-(3). 
In the RSFOPTSGEs (1)-(3), we take $T=2000$ (i.e., $0 \leq t \leq 2000$ ), $L=16,000$ (i.e., $0 \leq$ $x \leq 16,000), K=1, \alpha=1.5, \tau=h=0.01$, the boundary value function $g(t)=0.22$, and the initial function

$$
u(0, x)=\varphi(x)= \begin{cases}0.22+\sin (\pi x / 2000), & x \in[6000,8000] \\ 0.22, & x \in[0,600] \cup[8000,16,000]\end{cases}
$$

In this case, it is very difficult to find the analytical solution for RSFOPTSGEs, so that we can only find their numerical solutions. By the CNFD scheme we compute out the CNFD solution for $(t, x) \in[0,2000] \times[0,16,000]$ and depict it in Fig. 1 . They should coincide with the physical model rules.

In addition, according to Theorem 2, the theoretical errors of the CNFD solutions should be $O\left(\tau^{2}+h^{2}\right)=O\left(10^{-4}\right)$, whereas the numerical errors of the CNFD solutions are computed out by $\left\|\mathbf{U}^{n}-\mathbf{U}^{n-1}\right\|_{\infty}$ (due to $\left\|\mathbf{U}^{n}-\mathbf{U}^{n-1}\right\|_{\infty} \leq\left\|\mathbf{U}^{n}-\mathbf{U}_{a}^{n}\right\|_{\infty}+\left\|\mathbf{U}_{a}^{n}-\mathbf{U}_{a}^{n-1}\right\|_{\infty}+\| \mathbf{U}^{n-1}-$ $\left.\mathbf{U}_{a}^{n-1} \|_{\infty}\right)$, which are shown in Fig. 2 and can also attain $O\left(10^{-4}\right)$. This shows that the numerical conclusions coincide with theoretical results. Further, it is shown that the CNFD scheme is very efficient and feasible for solving the RSFOPTSGEs (1)-(3).
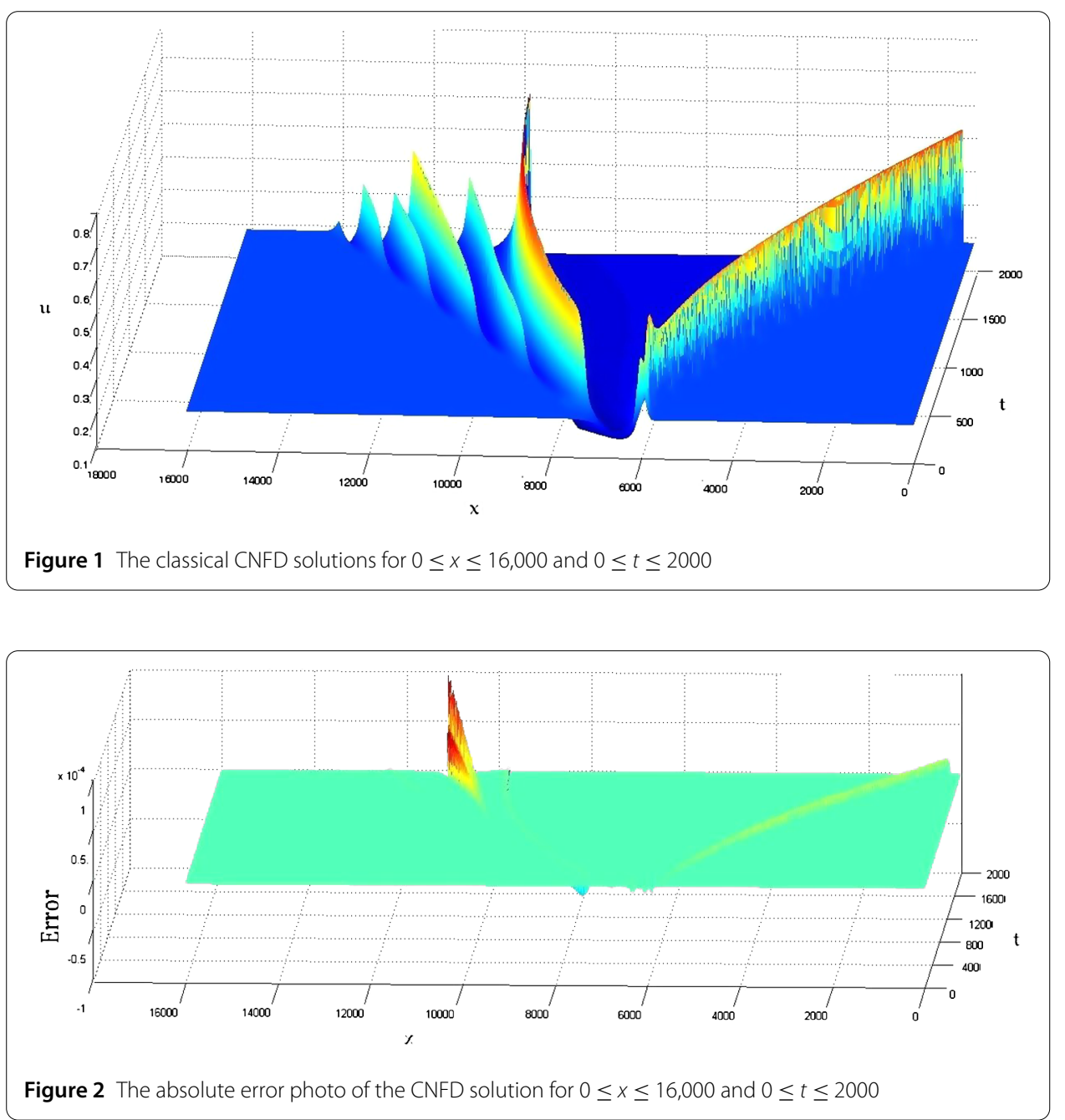


\section{Conclusions and discussion}

In this work, we have established the CNFD scheme for the RSFOPTSGEs (1)-(3) and analyzed the existence, uniqueness, stability, and convergence of the CNFD solutions. We have also used a numerical experiment to check the feasibility and effectiveness of the CNFD scheme and to verity that the numerical computing consequences are in accordance with theoretical analysis. Moreover, it is shown that the CNFD scheme is very valid and feasible for solving the RSFOPTSGEs (1)-(3).

Even if we only study CNFD scheme for the RSFOPTSGEs (1)-(3) in the one-dimensional space, the CNFD scheme can be easily and effectively used to solve the RSFOPTSGEs in two- and three-dimensional spaces.

\section{Acknowledgements}

The authors are thankful to the honorable reviewers and editors for their valuable suggestions and comments, which improved the paper.

\section{Funding}

This research was supported by the National Science Foundation of China grants 41704047 and 11671 106, the National Key Research and Development Project of China Grant 2017YFC1500301, and the cultivation fund of the National Natural and Social Science Foundations in BTBU Grant LKJJ2016-22.

\section{Availability of data and materials}

The authors declare that all data and material in the paper are available and veritable.

\section{Competing interests}

The authors declare that they have no competing interests.

\section{Authors' contributions}

Both authors contributed equally and significantly in writing this article. Both authors wrote, read, and approved the final manuscript.

\section{Author details}

${ }^{1}$ School of Science, Beijing Technology and Business University, Beijing, China. ${ }^{2}$ School of Mathematics and Physics, North China Electric Power University, Beijing, China.

\section{Publisher's Note}

Springer Nature remains neutral with regard to jurisdictional claims in published maps and institutional affiliations.

Received: 7 February 2018 Accepted: 6 June 2018 Published online: 22 June 2018

\section{References}

1. Kilbas, A.A., Srivastava, H.M., Trujillo, J.J.: Theory and Applications of Fractional Differential Equations. Elsevier, Amsterdam (2006)

2. Liu, F., Zhuang, P., Liu, Q.X.: Numerical Methods of Fractional Partial Differential Equations and Their Applications. Science Press, Beijing (2015) (in Chinese)

3. Elsaid, A., Hammad, D.: A reliable treatment of homotopy perturbation method for the sine-Gordon equation of arbitrary (fractional) order. J. Fract. Calc. Appl. 2(1), 1-8 (2012)

4. Yousef, A.M., Rida, S.Z., Ibrahim, H.R.: Approximate solution of fractional-order nonlinear sine-Gordon equation. Elixir Appl. Math. 82, 32549-32553 (2015)

5. Macías-Díaz, J.E.: Numerical study of the process of nonlinear supratransmission in Riesz space-fractional sine-Gordon equations. Commun. Nonlinear Sci. Numer. Simul. 46(1), 89-102 (2017)

6. Xia, H., Teng, F., Luo, Z.D.: A reduced-order extrapolating finite difference iterative scheme for 2D generalized nonlinear sine-Gordon equation. Appl. Comput. Math. 7(1), 19-25 (2018)

7. Batiha, B., Noorani, M.S.M., Hashim, I.: Approximate analytical solution of the coupled sine-Gordon equation using the variational iteration method. Phys. Scr. 76(5), 445-448 (2007)

8. Zhou, S.F.: Dimension of the global attractor for the damped sine-Gordon equation. Acta Math. Sin. 39(5), 597-601 (1996)

9. Liu, S., Fu, Z.T., Liu, S.: Exact solutions to sine-Gordon-type equations. Phys. Lett. A 351, 59-63 (2006)

10. Wazwaz, A.M.: Exact solutions for the generalized sine-Gordon and the generalized sinh-Gordon equations. Chaos Solitons Fractals 28, 127-135 (2006)

11. Zhang, W.S.: Finite Difference Methods for Partial Differential Equations in Science Computation. Higher Education Press, Beijing (2006) 